\title{
Sphingosine 1-phosphate levels in cerebrospinal fluid after subarachnoid hemorrhage
}

\author{
Anika Männer ${ }^{1}$, Dominique Thomas ${ }^{2}$, Marlies Wagner ${ }^{3}$, Jürgen Konczalla ${ }^{4}$, Helmuth Steinmetz ${ }^{1}$, \\ Robert Brunkhorst ${ }^{1,5}$ and Waltraud Pfeilschifter ${ }^{1 *}$ (i)
}

\begin{abstract}
Background and purpose: Sphingosin-1-phosphate (S1P) plays a crucial role as a signaling molecule in the immune system and the vasculature. Previous studies suggested a role as a vasoconstrictor of cerebral arteries via the S1P3Receptor. Cerebral vasospasm (VS) following aneurysmal subarachnoid hemorrhage (SAH) is a major cause of disability and poor neurological outcome. Early detection of vasospasm could facilitate the prevention of cerebral ischemia in SAH patients. The aim of this prospective case-control study was to characterize the dynamics of S1P in the cerebrospinal fluid (CSF) of patients with SAH in relation to hemorrhage volume, the occurrence of VS, and neurological outcome.
\end{abstract}

Methods: S1P levels in CSF of 18 control subjects and 18 SAH patients with placement of an external ventricular drainage (EVD) were determined by high sensitivity mass spectrometry from day 1 through 14 after SAH onset. Hemorrhage volume, development of asymptomatic vasospasm (aVS) and symptomatic vasospasm (sVS), and neurological outcome were correlated to day 1 S1P levels.

Results: The intrathecal S1P levels of SAH patients were higher than those of the control subjects, and correlated with hemorrhage volume. There was no significant difference in S1P levels between patients with aVS and those with sVS. S1P levels significantly correlated with neurological outcome on a sliding modified Rankin scale.

Conclusion: S1P levels were highest directly after placement of the EVD and correlated strongly with hemorrhage volume, which may be caused by the intrathecal clot and subsequent lysis of red blood cells, an important source of S1P. We did not detect a second peak of S1P release over the course of the intensive care period.

Keywords: Subarachnoid hemorrhage, Vasospasm, Delayed cerebral ischemia, Sphingosine 1-phosphate, Fingolimod, Neurological outcome

\section{Introduction}

Aneurysmal subarachnoid hemorrhage (SAH), accounting for approximately $5 \%$ of all strokes is a severe disease which is associated with a variety of primary and secondary complications [1]. Despite significant advances of neurointensive care, patient outcome remains poor in many cases. Only

\footnotetext{
* Correspondence: w.pfeilschifter@med.uni-frankfurt.de

${ }^{1}$ Frankfurt University Hospital, Department of Neurology, Goethe University,

Theodor-Stern-Kai 7, 60590 Frankfurt am Main, Germany

Full list of author information is available at the end of the article
}

about half of the patients (55\%) who survive the initial bleed recover functional independence in most activities of daily living after SAH [2]. Particularly the development of vasospasm (VS) and delayed cerebral ischemia (DCI) are strongly associated with poor functional outcome [3, 4]. According to Frontera et al., symptomatic vasospasm (sVS) was defined as worsening of clinical symptoms (new neurological signs or deterioration in level of consciousness) due to cerebral ischemia attributable to VS. [5] It is important to note that there are many other causes of clinical deterioration besides VS, such as infection, seizures, hypoxia, metabolic derangement, hydrocephalus, and others. Therefore, in

(c) The Author(s). 2020 Open Access This article is licensed under a Creative Commons Attribution 4.0 International License, which permits use, sharing, adaptation, distribution and reproduction in any medium or format, as long as you give appropriate credit to the original author(s) and the source, provide a link to the Creative Commons licence, and indicate if changes were made. The images or other third party material in this article are included in the article's Creative Commons licence, unless indicated otherwise in a credit line to the material. If material is not included in the article's Creative Commons licence and your intended use is not permitted by statutory regulation or exceeds the permitted use, you will need to obtain permission directly from the copyright holder. To view a copy of this licence, visit http://creativecommons.org/licenses/by/4.0/. 
2010 a consensus statement by a multidisciplinary group proposed a new definition for DCI and cerebral infarction after SAH [6], recommending a diagnosis of DCI only if the aforementioned other causes can be ruled out (by intensive clinical assessment, CT or MRI scan and appropriate laboratory examinations), and if the clinical deterioration persists for at least $1 \mathrm{~h}$ and does not appear immediately after aneurysm occlusion. Based on these hard criteria, the development of DCI is one of the most feared complications of $\mathrm{SAH}$ that can cause severe disability or even death [7]. Since many patients after severe SAH are comatose or sedated, the diagnosis of sVS and DCI is even more reliable if based on neuroimaging findings (CT or MRI).

Several clinical and imaging predictors of DCI after SAH have been identified, among them hemorrhage volume as graded on brain imaging with the modified Fisher score (mFS) [8]. The causative mechanisms for VS and DCI are yet unknown and there is no validated biomarker to identify patients at a high risk of VS and DCI after SAH.

Sphingosin 1-phosphate (S1P), a bioactive sphingolipid and signaling molecule, is involved in a wide variety of physiological and pathological processes, foremost in the immune system and the vasculature $[9,10]$. Its plasma levels are in the low micromolar range with red blood cells being the main source of plasma S1P, whereas its concentration in interstitial fluids is much lower [10]. Via this gradient, S1P regulates lymphocyte egress from the lymph nodes. The S1P pathway can be targeted pharmacologically in humans: the synthetic S1P analog FTY720 (fingolimod) has been marketed in 2010 as the first oral drug to prevent relapses in multiple sclerosis. Its presumed mechanisms of action are a reduction of circulating lymphocates but also direct brain specific effects [11]. There is evidence from preclinical studies that S1P is produced in the periinfarct cortex after cerebral ischemia [12], and preclinical studies as well as small clinical trials have shown neuroprotective effects of the functional S1P receptor antagonist fingolimod in ischemic stroke [13, 14]. In an experimental model of $\mathrm{SAH}$, fingolimod has been shown to reduce microvascular dysfunction and inflammation and improve neurological outcome of the animals [15]. Mechanistically, S1P has been proposed as putative mediator of VS as it has been shown to induce constriction of basilar arteries ex vivo via the S1P3 receptor [16]. S1P levels in CSF are low in healthy subjects $(<5 \mathrm{nM})$ and significantly elevated in early relapsing-remitting multiple sclerosis or infectious CNS diseases [17, 18]. After SAH, the lysis of red blood cells in SAH could lead to elevated S1P levels which in turn could contribute to VS and DCI.

The aim of this prospective case-control study was to characterize the dynamics of S1P levels in CSF of SAH patients over 14 days after symptom onset by high sensitivity mass spectrometry and to correlate them to hemorrhage volume, the occurrence of vasospasms and neurological outcome.

\section{Methods}

\section{Patients and clinical assessment}

The study was approved by the ethics committee of Frankfurt University Hospital (342/15). All consecutive SAH patients treated in our interdisciplinary neurointensive care unit from June 2016 to August 2017 were screened and informed consent was sought from patients or their legal representatives. We included patients $\geq 18$ years of age with nontraumatic aneurysmal SAH requiring an external ventricular drain (EVD) in the early management of hydrocephalus and elevated intracranial pressure. Exclusion criteria were pregnancy or previous neurological disorders (modified Rankin Scale $[\mathrm{mRS}] \geq$ 2). Samples of cerebrospinal fluid (CSF) were obtained from the EVD daily from day 1 to 14 .

Clinical outcome was determined at 12 month after discharge (mRS) (Table 1$)$. Control subjects $(n=18)$ were nonpregnant patients $\geq 18$ years of age who underwent lumbar puncture for the exclusion of SAH or inflammatory central nervous system (CNS) diseases and had normal CSF analysis.

Aneurysms were detected by digital subtraction catheter angiography (DSA). According to the assessment of an interdisciplinary neurovascular team, half of the patients were treated by occlusion of the aneurysm by neurosurgical clipping $(n=8)$, the other half of the patients were treated by endovascular occlusion by coiling $(n=8)$ (Table 1). All patients had aneurysm repair within the first $24 \mathrm{~h}$ after admission to the hospital and received the standard therapy for $\mathrm{SAH}$ in accordance to national and international guidelines: oral nimodipine $(360 \mathrm{mg} /$ day $)$, close monitoring of the mean arterial pressure (MAP), and the use of catecholamines to maintain a MAP of $60-90$ $\mathrm{mmHg}$ prior to detection of VS and $>100 \mathrm{mg}$ - if tolerated - after sonographic detection of VS.

Head CT was performed upon admission (in all cases within $24 \mathrm{~h}$ after symptom onset) and between 24 and $48 \mathrm{~h}$ after surgical or endovascular aneurysm repair (to examine the development of edema, secondary bleeding or new cerebral infarction) and before discharge. Additional brain imaging (CT mostly with $\mathrm{CT}$ angiography or MR with MR angiography) were performed as required when patients showed a clinical deterioration.

The development of vasospasm was monitored routinely by daily transcranial Doppler (TCD) ultrasonography. TCD vasospasm was defined as a mean flow velocity in the middle cerebral artery $(\mathrm{MCA}) \geq 120 \mathrm{~cm} / \mathrm{sec}$ [19]. In cases of vasospasm on TCD, conservative management was supplemented by induced hypertension (MAP $>100 \mathrm{mmHg}$ ). In most cases, vasospasm was confirmed by CT or MR angiography or digital subtraction angiography (DSA) (defined by $>1 / 3$ vasospastic arterial narrowing not attributable to atherosclerosis, catheter-induced spasm or vessel hypoplasia). 


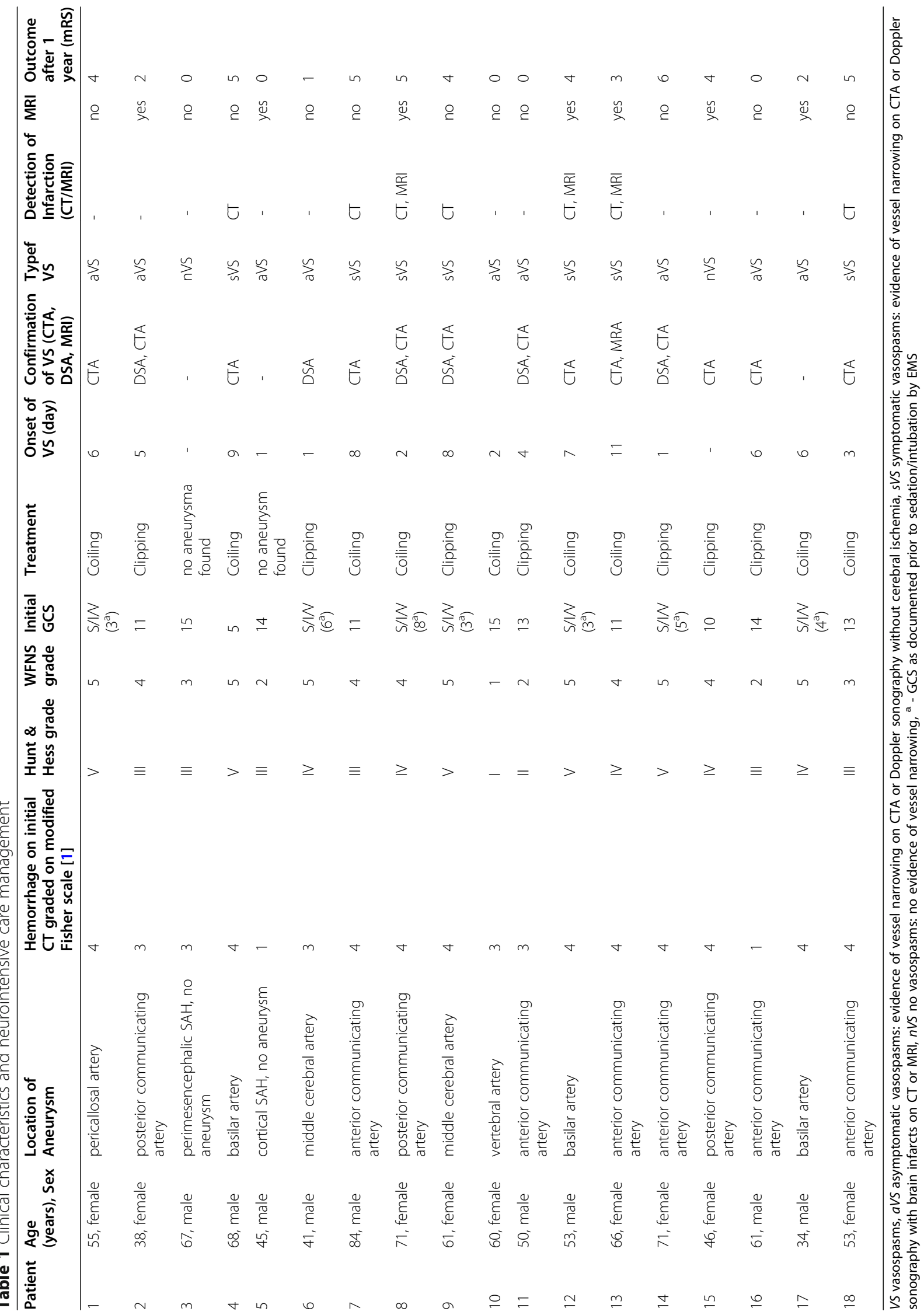


Because our patient cohort comprised mainly analgosedated patiens whose neurological function in terms of focal deficits could not be reliably assessed, we applied a strict definition of sVS: the documented vasospasm had to be compatible with new cerebral ischemia on clinically-indicated follow up brain imaging (28\% MRI, $72 \%$ CT) that had not been present on the imaging directly after aneurysm occlusion. Other causes of cerebral ischemia or DCI had to be excluded (re-bleeding, hydrocephalus and others).

Determination of sphingolipid concentrations by liquid chromatography/tandem mass spectrometry (LC-MS/MS) CSF samples were centrifuged at $1000 \times \mathrm{g}$ for $5 \mathrm{~min}$ at $4{ }^{\circ} \mathrm{C}$ and the supernatant stored at $-80^{\circ} \mathrm{C}$ until analysis. CSF samples $(100 \mu \mathrm{L})$ were mixed with $100 \mu \mathrm{L}$ buffer (citric acid $30 \mathrm{mM}$, disodium hydrogen phosphate $40 \mathrm{mM}$ ) and $20 \mu \mathrm{L}$ internal standard solution containing sphingosined7, sphinganine-d7 (30 ng/mL each), sphingosine-1phosphate-d7 (40 ng/mL, avanti polar lipids, Alabaster, USA). The mixture was extracted with $600 \mu \mathrm{L}$ methanol/ chloroform/hydrochloric acid (15:83:2, v/v/v). The organic phase was evaporated and reconstituted in $50 \mu \mathrm{L}$ of methanol.

Analytes were separated using an Agilent 1260 series binary pump (Agilent technologies, Waldbronn, Germany) equipped with a Kinetex EVO C18 column $(50 \mathrm{~mm} \times 2.1 \mathrm{~mm}, 1.7 \mu \mathrm{m}, 100 \AA$; Phenomenex, Aschaffenburg, Germany). Column temperature was $55^{\circ} \mathrm{C}$. Mobile phases were $0.5 \%$ formic acid and acetonitrile/ isopropanol/acetone (50:30:20, v/v/v) with $1 \%$ formic acid. A gradient program was used at a flow rate of 0.3 $\mathrm{mL} / \mathrm{min}$. Total running time was $7.5 \mathrm{~min}$. After every sample, ethanol was injected. The MS/MS analyses were performed in Multiple Reaction Monitoring (MRM) mode using a triple quadrupole mass spectrometer 5500QTRAP (Sciex, Darmstadt, Germany) operating in positive electrospray ionization mode.

Data Acquisition was performed using Analyst Software V 1.6 and quantification was performed with MultiQuant Software V 3.0 (Sciex, Darmstadt, Germany), employing isotope dilution mass spectrometry.

\section{Statistical analyses}

Statistical analyses were performed with Prism 7 (GraphPad Software, La Jolla, CA) and BiAS (Biometische Analyse von Stichproben, Epsilon Editorial). Results are given as mean \pm standard deviation (SD), the primary variable is the CSF-S1P concentration in $\mathrm{ng}$ / $\mathrm{ml}$. For all tests, a $p$ value of less than 0.05 was considered significant. All variables were tested for normal distribution by the Shapiro-Wilk Test and the respective statistic test method is detailed along with the results.

\section{Results}

\section{Patient characteristics}

Eighteen SAH patients (Table 1) were recruited and 18 control patients were matched. The two groups did not differ significantly in age or sex distribution (SAH group: mean age $57 \pm 13$ years, $50 \%$ females; controls: mean age $53 \pm 18$ years, $50 \%$ females). All SAH patients underwent aneurysm repair within $24 \mathrm{~h}$ of admission.

\section{S1P levels in CSF are elevated after SAH and correlate with hemorrhage volume}

S1P was detectable (threshold of detection $\leq 0.2 \mathrm{ng} / \mathrm{ml}$ or $0.53 \mathrm{nM}$ ) in the CSF of $9 / 18$ patients on day 1 after $\mathrm{SAH}$ and in additional 3 patients up to day 10 (Fig. 1). We did not find a significant difference in S1P levels in patients with neurosurgical in comparison to endovascular treatment. In the control cohort, CSF S1P levels were below the threshold of detection, representing a significant increase after SAH (Fisher's exact test at day $1, p=$ 0.000516) (Fig. 1). S1P levels in SAH patients were heterogeneous but showed a significant decrease after 14 days (Wilcoxon matched pairs test, $Z=2.3664, p=0.017960$ ). Fig. 2 illustrates the distribution of clinical characteristics in our patient cohort. In patients with large hemorrhage volume, indicated by modified Fisher Scale [20] $(\mathrm{mFS})=4$ $(11 / 18,61 \%)$ S1P levels were significantly higher than in $\mathrm{SAH}$ patients with $\mathrm{mFS} \leq 3$ (Wilcoxon Mann Whitney test, $\mathrm{Z}=2.150, p=0.031528$ ) (Fig. 2b). S1P levels at day 1 and $\mathrm{mFS}$ grade in the whole $\mathrm{SAH}$ patient cohort showed a significant correlation (Kendall Tau analysis, tau $\beta=0.502, p=0.029$, data not shown).

\section{Correlation of S1P levels in CSF with development of vasospasm and neurological outcome}

More than $50 \%$ of our patient cohort had a large hemorrhage volume $(\mathrm{mFS}=4)$ (Fig. 2a). 16/18 (88\%) patients developed VS (7/16 [44\%] sVS and 9/16 [56\%] asymptomatic vasospasm [aVS]). Symptomatic VS only occurred in patients with large hematomas $(\mathrm{mFS}=4)$. Although there was a trend towards higher S1P levels in patients with sVS, there was no significant difference between the mean S1P levels between groups with aVS and sVS (Wilcoxon Mann Whitney test, $\mathrm{Z}=1.470$, $p=0.141694$ ) (Fig. 2b). The onset of VS was between as early as day 1 and as late as day 8 . In the individual patients, there was no significant difference between the S1P level on day 1 and the level on the day of VS detection (Wilcoxon matched pairs test, $Z=1.3337, p=0.182315$, data not shown).

In a next step, S1P levels were correlated with neurological outcome at 12 months. We observed a significant correlation between the S1P level at day 1 and the mRS on a sliding scale (tau $ß=0.559, p=0.007$, data not shown). A dichotomized analysis of poor outcome $(\mathrm{mRS} \geq 4)$ and favourable outcome $(\mathrm{mRS} \leq 3)$ showed a trend of higher day $1 \mathrm{~S} 1 \mathrm{P}$ levels in patients with poor outcome without 


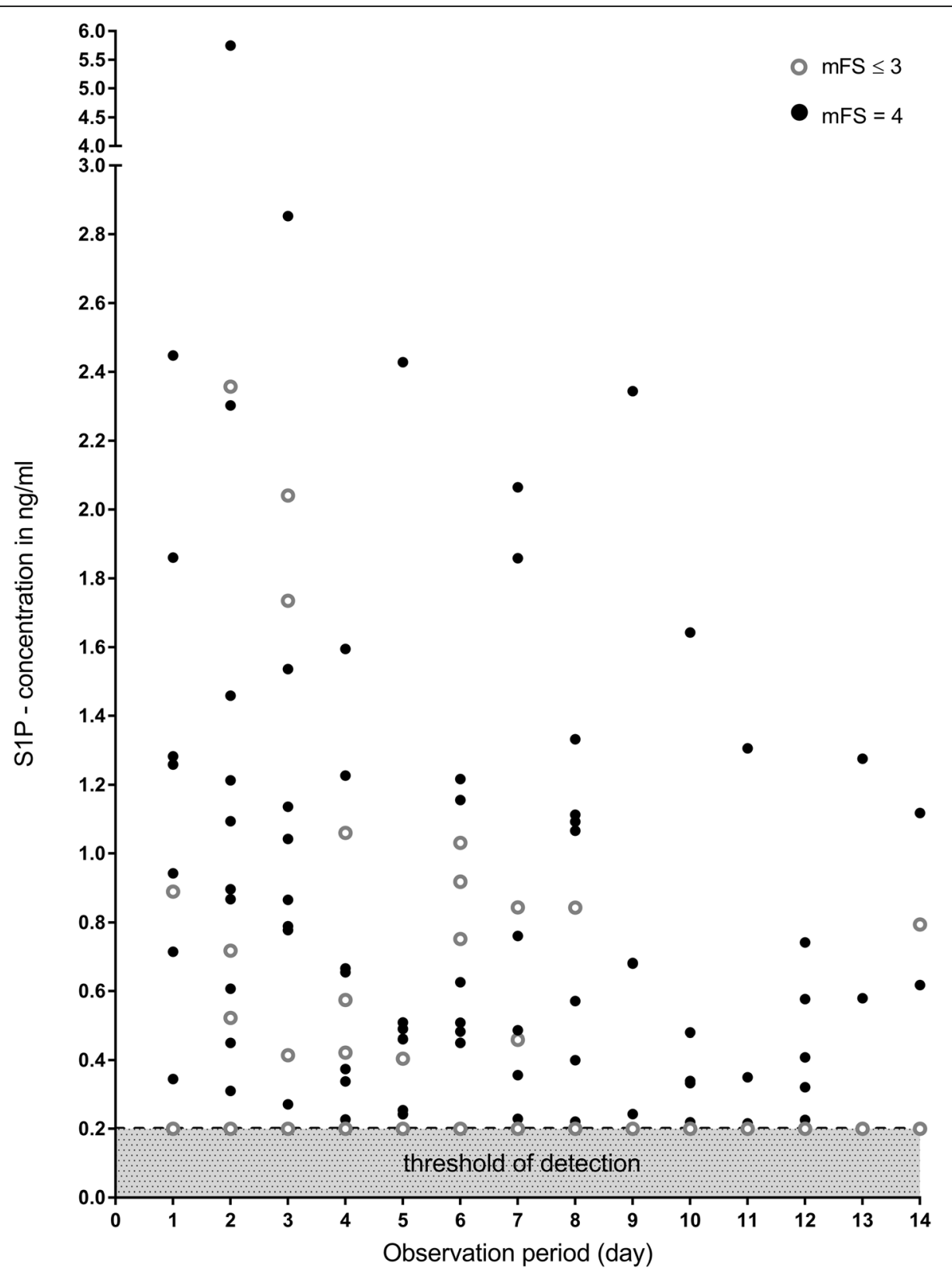

Fig. 1 S1P levels in CSF in SAH, measured at day 1 to day 14. S1P levels in CSF were measured with high sensitivity tandem mass spectrometry. Only values above the threshold of detection $(0.2 \mathrm{ng} / \mathrm{ml}=0.52 \mathrm{nM})$ are depicted. S1P levels of all control patients were below the threshold of detection

reaching statistical significance (Wilcoxon Mann Whitney test, $\mathrm{Z}=1.673, p=0.094264$ ) (Fig. $2 \mathrm{~b}$ ).

\section{Discussion}

In this prospective case-control study of S1P levels in the CSF of SAH patients over 14 days, we found that contrary to healthy controls, whose S1P levels were below the threshold of detection, S1P was detectable in the low nanomolar range in half of the patients, thus representing a significant increase after SAH. Owing to the significant correlation of S1P levels with hemorrhage volume and to the temporal profile with decreasing S1P levels over the course of 14 days, we assume that the increase in intrathecal S1P can mainly be attributed to the hemorrhage and subsequent lysis of red blood cells. This was also supported by the lack of a temporal association between S1P levels and the occurrence of vasospasm or new cerebral infarction. By contrast, a secondary intrathecal production, e.g. by invading immune cells or release from ischemic brain tissue, does not seem to play a relevant role since in this case secondary increases of S1P levels would have to be expected. The significant correlation between S1P levels and 


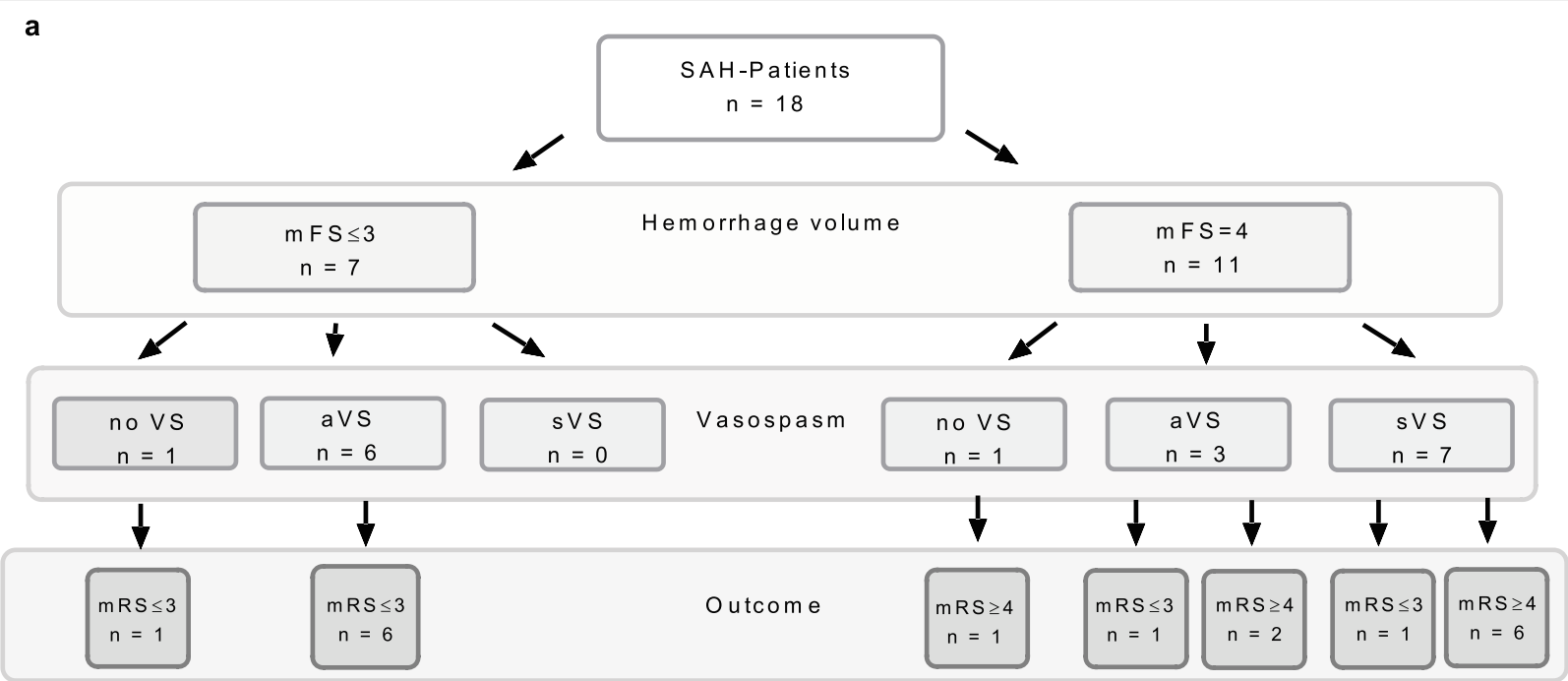

b
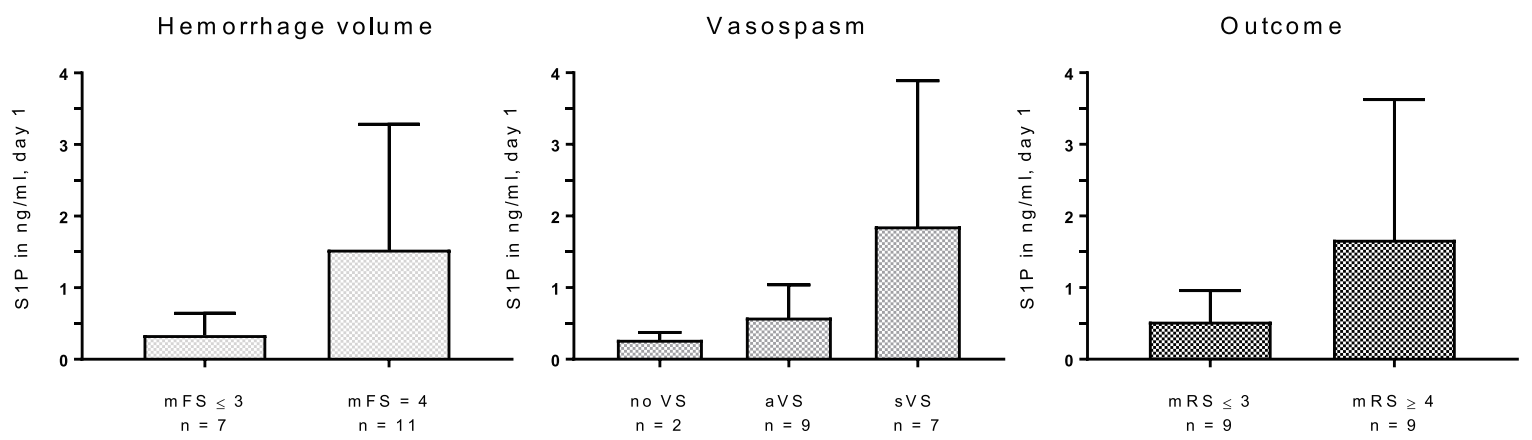

Fig. 2 a Hemorrhage volume, detection of vasospasm and functional outcome at 90 days in the SAH patient cohort. mFS = modified Fisher scale, $n V S=$ no vasospasm, aVS = asymptomatic vasospasm, sVS = symptomatic vasospasm, mRS = modified Rankin scale score. $\mathbf{b}$ Correlation of S1P levels in CSF with dichotomized hemorrhage volume, occurrence of vasospasm and neurological outcome. Bars represent the means \pm SD. mFS - modified Fisher Scale, sVS - symptomatic vasospasm, aVS - asymptomatic vasospasm, mRS - modified Rankin scale score

neurological outcome on a sliding mRS scale could well reflect the known influence of hemorrhage volume on outcome after SAH, so that our study does not yet justify the assumption of an independent role of CSF-S1P as a predictive biomarker in SAH. Our data do not exclude that S1P contributes to VS after SAH, which would be pathophysiologically conceivable since S1P has been shown to act as a vasoconstrictor on cerebral arteries [16] and treatment of experimental animals with the functional S1P receptor antagonist fingolimod has been shown to ameliorate the sequelae of SAH [15]. Nevertheless, the relatively low CSF concentrations after SAH found in our study do not encourage e.g. the intrathecal scavenging of this mediator with a neutralizing antibody as it has been proposed for other diseases [21].

To the best of our knowledge, this is the first prospective study investigating the dynamics of S1P levels in CSF after SAH. S1P is a dynamic and instable lipid mediator and requires careful preanalytical processing [22]. Hence, in a previous investigation of sphingolipid profiles in the CSF of patients at a single time point post SAH, the S1P concentration was below the limit of detection and did not allow correlation with clinical parameters [23].

A strength of our study is the daily sampling over 14 days which allowed to characterize the temporal dynamics of intrathecal S1P after SAH and excluded a secondary increase during the VS phase but the limitation of a relatively small sample size, especially in view of the large variability of the S1P concentrations, has to be taken into account.

\section{Acknowledgements}

We gratefully acknowledge the willingness to participate of the patients and their legal representatives as well as the support of the Neuro-ICU nursing team of Frankfurt University Hospital. 


\section{Authors' contributions}

$\mathrm{AM}, \mathrm{RB}$ and WP conceived the study. AM collected patient data and CSF samples. AM and RB managed the Data. AM, RB and WP performed statistical analysis and wrote a first draft of the manuscript. AW, JK and HS contributed significantly to the design of the study and revised the manuscript providing important intellectual content. The author(s) read and approved the final manuscript.

\section{Funding}

$\mathrm{RB}$ and WP received funding from the Fondation Leducq (SphingoNet) and the German Research Foundation (SFB 1039/TP B08).

\section{Availability of data and materials}

The dataset of the current study is available from the corresponding author upon reasonable request.

\section{Ethics approval and consent to participate}

The study was approved by the ethics committee of Frankfurt University Hospital (342/15) and consent to participate was obtained from patients or their legal representatives.

\section{Consent for publication}

The consent by patients or their legal representatives included the consent for publication of anonymized data.

\section{Competing interests}

The authors do not have competing interests.

\section{Author details}

${ }^{1}$ Frankfurt University Hospital, Department of Neurology, Goethe University, Theodor-Stern-Kai 7, 60590 Frankfurt am Main, Germany. ${ }^{2}$ Pharmazentrum Frankfurt, Frankfurt University Hospital, Department of Clinical Pharmacology, Goethe University, Theodor-Stern-Kai 7, 60590 Frankfurt am Main, Germany. ${ }^{3}$ Frankfurt University Hospital, Institute for Diagnostic and Interventional Neuroradiology, Goethe University, Theodor-Stern-Kai 7, 60590 Frankfurt am Main, Germany. ${ }^{4}$ Frankfurt University Hospital, Department of Neurosurgery, Goethe University, Theodor-Stern-Kai 7, 60590 Frankfurt am Main, Germany. ${ }^{5}$ RWTH Uniklinik Aachen, Klinik für Neurologie, Pauwelsstraße 30, 52074 Aachen, Germany.

Received: 23 October 2020 Accepted: 29 October 2020

Published online: 23 November 2020

\section{References}

1. Ingall, T., Asplund, K., Mahonen, M., \& Bonita, R. (2000). A multinational comparison of subarachnoid hemorrhage epidemiology in the WHO MONICA stroke study. Stroke, 31, 1054-1061 5.

2. Nieuwkamp, D. J., Setz, L. E., Algra, A., Linn, F. H. H., de Rooij, N. K., \& Gabriël, J. E. (2009). Changes in case fatality of aneurysmal subarachnoid haemorrhage over time, according to age, sex, and region: A meta-analysis. Lancet Neurology, 8, 635.

3. Bederson, J. B., Levy, A. L., Ding, W. H., et al. (1998). Acute vasoconstriction after subarachnoid hemorrhage. Neurosurgery, 42, 352-360.

4. Vergouwen, M. D. I., Etminan, N., llodigwe, D., \& Macdonald, R. L. (2011). Lower incidence of cerebral infarction correlates with improved functional outcome after aneurysmal subarachnoid hemorrhage. Journal of Cerebral Blood Flow and Metabolism, 31, 1545-1553.

5. Frontera, J. A., Fernandez, A., Schmidt, J. M., Claassen, J., Wartenberg, K. E., Badjatia, N., ... Mayer, S. A. (2009). Defining vasospasm after Sucharachnoid hemorrhage, what is the most clinically relevant definition? Stroke, 40, 1963-1968.

6. Vergouwen, M. D. I., Vermeulen, M., Van Gijn, J., Rinkel, G. J. E., Wijdicks, E. F., et al. (2010). Definition of delayed cerebral ischemia after aneurysmal subarachnoid hemorrhage as an outcome event in clinical trials and observational studies. Stroke, 41, 2391-2395.

7. Rabinstein, A. A., Friedman, J. A., Weigand, S. D., McClelland, R. L., Fulgham, J. R., Manno, E. M., ... Wijdicks, E. F. (2004). Predictors of cerebral infarction in aneurysmal subarachnoid hemorrhage. Stroke, 35, 1862-1866.

8. Lee, H., Perry, J. J., English, S. W., Alkherayf, F., Joseph, J., Nobile, S., et al. (2018). Clinical prediction of delayed cerebral ischemia in aneurysmal subarachnoid hemorrhage. Journal of Neurosurgery, 8, 1-8.
9. Maceyka, M., \& Spiegel, S. (2014). Sphingolipid metabolites in inflammatory disease. Nature, 510, 58-67.

10. Obinata, H., \& Hla, T. (2012). Sphingosine 1-phosphate in coagulation and inflammation. Semin Immunpathol, 34(1), 73-91.

11. Choi, J. W., Gardell, S. E., Herr, D. R., Rivera, R., Lee, C. W., Noguchi, K., et al. (2011). FTY720 (fingolimod) efficacy in an animal model of multiple sclerosis requires astrocyte sphingosine 1-phosphate receptor 1 (S1P1) modulation. Proceedings of the National Academy of Sciences of the United States of America, 108, 751-756.

12. Brunkhorst, R., Kanaan, N., Koch, A., Ferreirós, N., Mirceska, A., Zeiner, P., et al. (2013 Jul 31). FTY720 treatment in the convalescence period improves functional recovery and reduces reactive astrogliosis in photothrombotic stroke. PLoS One, 8(7), e70124.

13. Kraft, P., Göb, E., Schuhmann, M. K., Göbel, K., Deppermann, C., Thielmann, l., et al. (2013). FTY720 ameliorates acute ischemic stroke in mice by reducing thromboinflammation but not by direct neuroprotection. Stroke., 44, 3202-3210.

14. Fu, Y., Zhang, N., Ren, L., Yan, Y., Sun, N., Li, Y. J., et al. (2014). Impact of an immune modulator fingolimod on acute ischemic stroke. Proceedings of the National Academy of Sciences of the United States of America, 111, 18315-18320.

15. Xu, H. L., Pelligrino, D. A., Paisansathan, C., \& Testai, F. D. (2015). Protective role of fingolimod (FTY720) in rats subjected to subarachnoid hemorrhage. Journal of Neuroinflammation, 12, 16.

16. Salomone, S., Potts, E. M., Tyndall, S., Ip, P. C., Chun, J., Brinkmann, V., et al. (2008). Analysis of sphingosine 1-phosphate receptors involved in constriction of isolated cerebral arteries with receptor null mice and pharmacological tools. British Journal of Pharmacology, 153, 140-147.

17. Kułakowska, A., Zendzian-Piotrowska, M., Baranowski, M., Konończuk, T., Drozdowski, W., Górski, J., et al. (2010). Intrathecal increase of sphingosine 1phosphate at early stage multiple sclerosis. Neuroscience Letters, 477, 149-152.

18. Kułakowska, A., Byfield, F. J., Zendzian-Piotrowska, M., Zajkowska, J. M. Drozdowski, W., Mroczko, B., ... Bucki, R. (2014). Increased levels of sphingosine-1-phosphate in cerebrospinal fluid of patients diagnosed with tick-borne encephalitis. Journal of Neuroinflammation, 11, 193.

19. Vora, Y. Y., Suarez-Almazor, M., Steinke, D. E., Martin, M. L., \& Findlay, J. M. (1999). Role of transcranial Doppler monitoring in the diagnosis of cerebral vasospasm after subarachnoid hemorrhage. Neurosurgery, 44, 1237-1247.

20. Frontera, J. A., Claassen, J., Schmidt, J. M., Wartenberg, K. E., Temes, R., Conolly Jr., E. S., et al. (2006). Prediction of symptomatic vasospasm after subarachnoid hemorrhage: The modified fisher scale. Neurosurgery., 59, 21-27.

21. Pal, S. K., Drabkin, H. A., Reeves, J. A., Hainsworth, J. D., Hazel, S. E., Paggiarino, D. A., et al. (2017). A phase 2 study of the sphingosine-1phosphate antibody sonepcizumab in patients with metastatic renal cell carcinoma. Cancer., 123, 576-582.

22. Brunkhorst R, Pfeilschifter W, Patyna S, Büttner S, Eckes T, Trautmann S, et al. (2018). Preanalytical Biases in the Measurement of Human Blood Sphingolipids. Int J Mol Sci., 19, (5):1390.

23. Testai, F. D., Xu, H. L., Kilkus, J., Suryadevara, V., Gorshkova, I., Berdyshev, E., ... Dawson, G. (2015). Changes in the metabolism of sphingolipids after subarachnoid hemorrhage. Journal of Neuroscience Research, 93, 796-805.

\section{Publisher's Note}

Springer Nature remains neutral with regard to jurisdictional claims in published maps and institutional affiliations.

Ready to submit your research? Choose BMC and benefit from:

- fast, convenient online submission

- thorough peer review by experienced researchers in your field

- rapid publication on acceptance

- support for research data, including large and complex data types

- gold Open Access which fosters wider collaboration and increased citations

- maximum visibility for your research: over $100 \mathrm{M}$ website views per year

At $\mathrm{BMC}$, research is always in progress.

Learn more biomedcentral.com/submissions 